\title{
Effects of tDCS-induced Motor Cortex Modulation on Pain in HTLV-1
}

\author{
A Blind Randomized Clinical Trial
}

\author{
Giórgio Souto, PT, MSc,* Igor C. Borges, MDS, † Bruno T. Goes, PT, MSc, ${ }^{*}$ \\ Mariana E. de Mendonça, PT,ł Roberta G. Gonçalves, PT, $\dagger$ Lucas B. Garcia, PTS, $\dagger$ \\ Katia N. Sá, PT, PhD, ${ }^{*} \dagger$ Márcio R. Coutinho, BDS, PhD, $\dagger$ Bernardo Galvão-Castro, MD, PhD,* \\ Felipe Fregni, MD, PhD, $\S$ and Abrahão F. Baptista, PT, PhD* $\dagger$
}

\begin{abstract}
Objective: We aimed to evaluate the effects of transcranial direct current stimulation (tDCS) on chronic pain in human T-lymphotropic virus type I-infected patients.
\end{abstract}

Materials and Methods: This is a sham-controlled randomized clinical trial. Twenty participants were randomized to receive active or sham anodal tDCS over the primary motor cortex (M1), with $2 \mathrm{~mA}, 25 \mathrm{~cm}^{2}$ electrodes, for 20 minutes on 5 consecutive days. Pain intensity was measured at baseline and after each day of treatment using a Visual Analog Scale. Associated factors such as pain components description, pressure pain threshold, and Timed Up and Go task were also assessed.

Results: Mild adverse events were reported by $100 \%$ of patients in the tDCS group and $90 \%$ in the sham group. Comparison of daily Visual Analog Scale pain scores from both groups demonstrated a significant effect for the factor Time $(P<0.001)$, but not for Group $(P=0.13)$ or Time $\times$ Group interaction $(P=0.06)$. There were 8 $(80 \%)$ responders (reduction of $50 \%$ or more in pain intensity) in the tDCS group and $3(30 \%)$ in the sham group $(P=0.03)$. Both groups demonstrated improvements for most associated factors evaluated. However, there was no difference in between-groups comparison analyses.

Conclusions: The analysis of the main outcomes in this study did not demonstrate a significant advantage of anodal tDCS applied to M1 in patients with human T-lymphotropic virus type I and chronic pain in comparison with sham tDCS, although secondary analysis suggests some superiority of active tDCS over sham. The large placebo effect observed in this study may explain the small differences between sham versus active tDCS.

Key Words: chronic pain, HTLV-1, randomized clinical trial, tDCS (Clin J Pain 2014;30:809-815)

Received for publication December 28, 2012; revised December 2, 2013; accepted September 11, 2013.

From the *Bahian School of Medicine and Public Health; $\dagger$ Laboratory of Functional Electrostimulation, Federal University of Bahia, Salvador, Bahia; \$Department of Neuroscience and Behavior, Institute of Psychiatry, University of São Paulo, São Paulo, Brazil; and §Laboratory of Neuromodulation, Spaulding Rehabilitation Hospital, Harvard Medical School, Boston, MA.

G.S.G. was funded by FAPESB, Salvador, BA/Brazil (Fundação de Amparo a Pesquisa do Estado da Bahia) and M.E.M by CAPES, Brasília, DF/Brazil (Coordenação de Aperfeiçoamento Pessoal de Nivel Superior). The authors declare no conflict of interest.

Reprints: Abrahão F. Baptista, PT, PhD, Laboratório de Eletroestimulação Funcional, Instituto de Ciências da Saúde, UFBA, Avenida Reitor Miguel Calmon, S/N, Vale do Canela, CEP: 40.110902, Salvador, Bahia, Brasil (e-mail: afbaptista@ufba.br).

Copyright (C) 2013 by Lippincott Williams \& Wilkins
$\mathrm{H}$ uman T-lymphotropic virus type I (HTLV-1) is a retrovirus that causes a chronic infectious disease and is associated with progressive neurological damage. ${ }^{1}$ Some patients infected with this virus present motor, sensory, and autonomic functional deficits. ${ }^{2-5}$ Among the clinical manifestations of this disease, chronic pain has been frequently observed. ${ }^{6-8}$ One study found that $>84.5 \%$ of HTLV-1infected patients reported chronic pain, having an incapacitating effect in most of the cases., 79

Although the mechanisms of pain in HTLV-1 are not fully elucidated, current evidence suggests that spinal cord degeneration may lead to secondary central sensitization. . $^{6,8}$ Given the central changes associated with this condition, current analgesic therapies commonly fail to provide sufficient relief for chronic pain. ${ }^{10}$ Therefore, new alternatives for pain relief using targeted central methods of neuromodulation may be desirable due to its central effects. ${ }^{11-14}$

Transcranial direct current stimulation (tDCS) has shown promising results in inducing cortical plasticity with clinical benefits in neuropathic and nociceptive pain settings. ${ }^{15-18}$ In tDCS, a weak electric current is delivered to the cerebral cortex through the scalp. ${ }^{19}$ The exposure of neural cells to a constant monophasic electric current induces modifications in their membrane excitability, resulting in local and distant plastic changes. ${ }^{12,20-24}$ Although a single session of tDCS is associated with transient effects in cortical excitability, it has been shown that daily sessions of anodal tDCS can induce longer-lasting effects. ${ }^{11,17,25}$ Nevertheless, although the initial trials show general positive results, there is limited evidence of the analgesic effect of tDCS applied to the primary motor cortex (M1). ${ }^{26}$

We aimed to assess the effects of tDCS on pain intensity, and secondarily on pain characteristics and motor function, in HTLV-1-infected patients. We hypothesized that 5 days of active tDCS to the M1 would result in significant changes in pain intensity when compared with patients receiving sham treatment.

\section{MATERIALS AND METHODS}

\section{Study Population}

The study consisted of a randomized, sham-controlled clinical trial conducted at the Reference Center for the Integrated and Multidisciplinary Treatment for HTLV-1 and Viral Hepatitis of the Bahian School of Medicine and Public Health (EBMSP). The study included HTLV-1infected patients, aged 18 years and older, with stable 
pharmacotherapy in the preceding month and with chronic low back and/or lower limbs pain. Chronic pain was defined as pain reported at least 4 days in a week at the same site, lasting $>6$ months and with intensity $\geq 4$ on a 10-point Visual Analog Scale (VAS), in which $0=$ "no pain" and $10=$ "worst possible pain." Individuals with cognitive impairment; a metal implant in the head; history of drug abuse; significant heart, lung, kidney, or skin disease; thyroid disorders; or women who were pregnant were excluded.

The study was approved by the Research Ethics Committee of the EBMSP, No. 238/2010, in agreement with Resolution 196/96 of the National Health Council (Brazil). Written informed consent was obtained from all participants.

\section{Randomization}

Participants were randomized using a stratified randomization strategy with pain as the stratification factor. To ensure that pain levels would be similar between the 2 groups, patients were allocated according to pain intensity at baseline. Participants were divided into 2 strata using a pain intensity cutoff of $7 / 10$ (VAS). A previously generated randomization list was used to allocate the patients to each stratum, in accordance with the order of their entrance into the study. A researcher who was not involved with assessments or interaction with participants randomized and allocated the patients (M.E.M.).

\section{Assessment Methods and Instruments}

Blinded researchers performed assessments (G.S.G., L.B.G.). Pain evaluation was restricted to the most important pain site reported. Pain intensity was assessed using a 10-point VAS, and the pain components were discriminated using the Brazilian version of the Short Form McGill Pain Questionnaire. ${ }^{27}$ Type of pain (neuropathic or nociceptive) was evaluated using the Brazilian version of the DN4 questionnaire (Douleur Neurophatique 4). ${ }^{28}$ Pressure pain threshold (PPT) was assessed with a pressure algometer (Pain Diagnostic \& Thermographics Great Neck, New York). ${ }^{29}$ For this evaluation, patients were comfortably, with both feet on the floor, and pressure was applied bilaterally to the tibial tuberosity at the rate of $1 \mathrm{~kg} / \mathrm{s}$ until the minimum pressure to trigger pain was reached. Two measures were taken, one to each tibial tuberosity, and the average of this was used in the final analysis. This specific measure aimed to determine whether there was a systemic analgesic effect as a result of the treatment protocol. Gait performance was evaluated by the Timed Up and Go (TUG) task. ${ }^{30}$ Quality of life was assessed using the SF-36 inventory, ${ }^{31}$ and depression using the first module of the Mini International Neuropsychiatric Interview (M.I.N.I). ${ }^{32}$ Data regarding demographic characteristics, type of pain (DN4), quality of life, and depression were collected only at baseline to assess whether the groups-tDCS and shamwere comparable in regard to significant variables in patients' pain, and because the instruments were not sensitive to detect changes after a 1-week period.

\section{Intervention-tDCS}

Trained researchers (B.T.G., I.C.B., and R.G.G.) conducted the stimulation protocol. For the treatment procedures, we used a tDCS montage that has been extensively used: an anode was placed over the C 3 position, corresponding in the International 10/20 EEG System of Electrode Placement ${ }^{33}$ to the left $\mathrm{M} 1$, and the cathode over the right supraorbital area. The stimulation was performed using pairs of silicone-sponge surface electrodes, and a clinical electro-stimulator (Striat, Ibramed, Brazil).

Most of the patients in this study had an AfroAmerican hair type, causing difficulties in exposing the skin during the application of tDCS, and so a $5 \times 5 \mathrm{~cm}$ electrode was chosen to increase the current density and to provide more effective stimulation. ${ }^{34,35}$ The current was delivered at $2 \mathrm{~mA}$ for $20 \mathrm{~min} / \mathrm{d}$, for 5 consecutive days. Current was gradually increased on the first 30 seconds and decreased on a similar pattern at the end of the stimulation.

For the sham group, the same parameters were used, but the current was only turned on for the initial $30 \mathrm{sec}-$ onds. This treatment was also performed for $20 \mathrm{~min} / \mathrm{d}$ for 5 consecutive days. The monitoring of possible adverse effects was conducted by the application of a tDCS adverse effects questionnaire after each treatment session.

\section{Statistical Analysis}

The sample size calculation was based on a previous study. ${ }^{15}$ An estimation was performed by considering a repeated-measures analysis of variance (ANOVA) between factor comparison, using $F$-value of $61.1, \alpha$-value of $5 \%$, study power of $80 \%, 2$ groups, 6 measures, and correction between repeated measures of 0.5 . The sample size estimation was 16 individuals -8 per group $\left(G^{*}\right.$ Power 3 , Germany). We decided to use 10 individuals per group to prevent losses on the follow-up.

Data were analyzed according to their distribution and was presented in terms of central tendency and dispersion (mean $\pm \mathrm{SD}$ or median [25th to 75th quartiles]). The comparisons between baseline characteristics of tDCS and sham groups were performed using the $\chi^{2}$ or Fisher exact test for the categorical variables. For continuous variables (PPT, TUG, and McGill), parametric or nonparametric tests were used after testing for normality (paired - paired $t$ test or Wilcoxon; unpaired - unpaired $t$ test or Mann-Whitney). The evaluation of pain intensity over the days, within both groups, and between the treatment groups, was performed using repeatedmeasures ANOVA. The dependent variable in this test was pain intensity and the main factors were Group (stimulation vs. sham), Time (at baseline and after each day of stimulation), and Group $\times$ Time, followed by a post hoc test with Bonferroni correction. The between-groups comparison of repeatedmeasures ANOVA was the statistical analysis used to evaluate the main effect of the primary outcome of the study. For the assessment of each group separately, the repeated-measures ANOVA was performed with pain intensity as a dependent variable and Time as the main factor, followed by a post hoc test using the Bonferroni correction. Neuropathic pain patients were also evaluated in a separate analysis, to consider if there was a different pain behavior in this group. $\alpha$-value was established at $5 \%(P<0.05)$ with a study power of $80 \%$. Finally, separate analyses comparing responders to nonresponders were carried out using cutoffs of $30 \%$ and $50 \%$ reduction in pain intensity. All the randomized patients were analyzed according to the randomization assignment (intention-to-treat analysis). We used the method of last observation carried forward. Data analysis was performed using the Statistical Package SPSS (version 19.0) by 2 blinded researchers (A.F.B. and F.F.).

\section{RESULTS}

Twenty individuals were initially included in the study and 10 patients were randomized to each study group. Two 
patients from the sham group were withdrawn from the study after the first and second session of treatment due to protocol violation (the regimen of analgesic used was changed - both patients reported the use of corticosteroids due to an increase in pain) (Fig. 1). Data regarding VAS pain scores was analyzed using the principle of intention-totreat analysis (last observation carried forward). This proceeding was not considered for TUG, McGill, and PPT, as these measurements were only performed on the first and last days of treatment. The sociodemographic, clinical, and baseline characteristics of the studied groups are shown in Table 1.

Adverse events (AEs) were reported in $10(100 \%)$ patients in the tDCS group and $9(90 \%)$ patients in the sham group. Among the patients reporting in at least 1 session of treatment, the average number of days in which an adverse effect was registered for each patient was 3.3 in the tDCS group and 2.9 in the sham group. Table 2 shows the frequency of each AE reported. The majority of these symptoms began in the first 30 seconds of intervention, and lasted from a few seconds to a few minutes. In all cases, the intensity of these symptoms was reported to be mild and tolerable.

Evaluation of daily VAS pain intensity scores from both groups using repeated-measures ANOVA demonstrated a significant effect for Time $\left(F_{2.71,48.86}=18.55\right.$, $P<0.001)$, but not an effect for Time $\times$ Group interaction $\left(F_{2.71,48.86}=2.68, \quad P=0.06\right) \quad$ or Group $\left(F_{1,18}=2.55\right.$, $P=0.13)$. Mean VAS scores at baseline and after each day of intervention are depicted in Figure 2, and also presented in Table 3.

When variances were compared for each isolated group (tDCS and sham), repeated-measures ANOVA of VAS pain scores in the tDCS group determined a significant effect for Time $\left(F_{1.93,17.37}=13.2, P<0.001\right)$ within subjects. Post hoc analysis revealed that VAS pain scores from the second, fourth, and fifth days of stimulation differed from baseline (mean difference from baseline VAS scores: D1 0.62, $P=0.3 ; \mathrm{D} 2$ 3.31, $P=0.03$; D3 3.03, $P=0.13$; D4 4.28, $P=0.006$; D5 3.57, $P=0.03$ ). The sham group also demonstrated a significant effect for Time $\left(F_{2.6,23.5}=6.33, P=0.004\right)$ for the same analysis. Post hoc tests showed a significant difference from baseline pain intensity on the fourth and fifth day of treatment (mean difference from baseline VAS scores: D1 1.09, $P=0.5$; D2 $1.29, P=1$; D3 2.05, $P=0.3$; D4 2.49, $P=0.02$; D5 2.28, $P=0.02$ ).

Considering only patients with neuropathic pain from both treatment groups, the evaluation of daily VAS pain scores by repeated-measures ANOVA demonstrated a significant effect for the factor Time $\left(F_{2.62,28.85}=12.19\right.$, $P<0.001)$, but not for Time $\times$ Group $\left(F_{2.62,28.85}=1.18\right.$, $P=0.3)$ or Group $\left(F_{1,11}=0.29, P=0.6\right)$ factors. The between-groups comparison of mean VAS pain scores at baseline and after each day of intervention, among patients with neuropathic pain, is depicted in Figure 3.

Repeated-measures ANOVA of VAS pain scores from patients with neuropathic pain in the tDCS group showed a significant effect for the factor Time $\left(F_{18,8.98}=5.32\right.$, $P=0.03$ ) within subjects. Post hoc tests did not demonstrate any statistically significant difference between baseline VAS pain scores and the consecutive days of intervention (mean difference from baseline VAS scores: D1 $0.45, P=1$ D2 2.93, $P=0.8$; D3 2.65, $P=1$; D4 4.53, $P=0.2$; D5 3.3, $P=0.9$ ). Patients with neuropathic pain from the sham group also demonstrated a significant effect for the factor Time $\left(F_{2.64,15.82}=9.06, P=0.001\right)$ for the same analysis of VAS scores. Post hoc tests demonstrated a statistically significant difference between baseline pain intensity and pain intensity on D2, D4, and D5 (mean

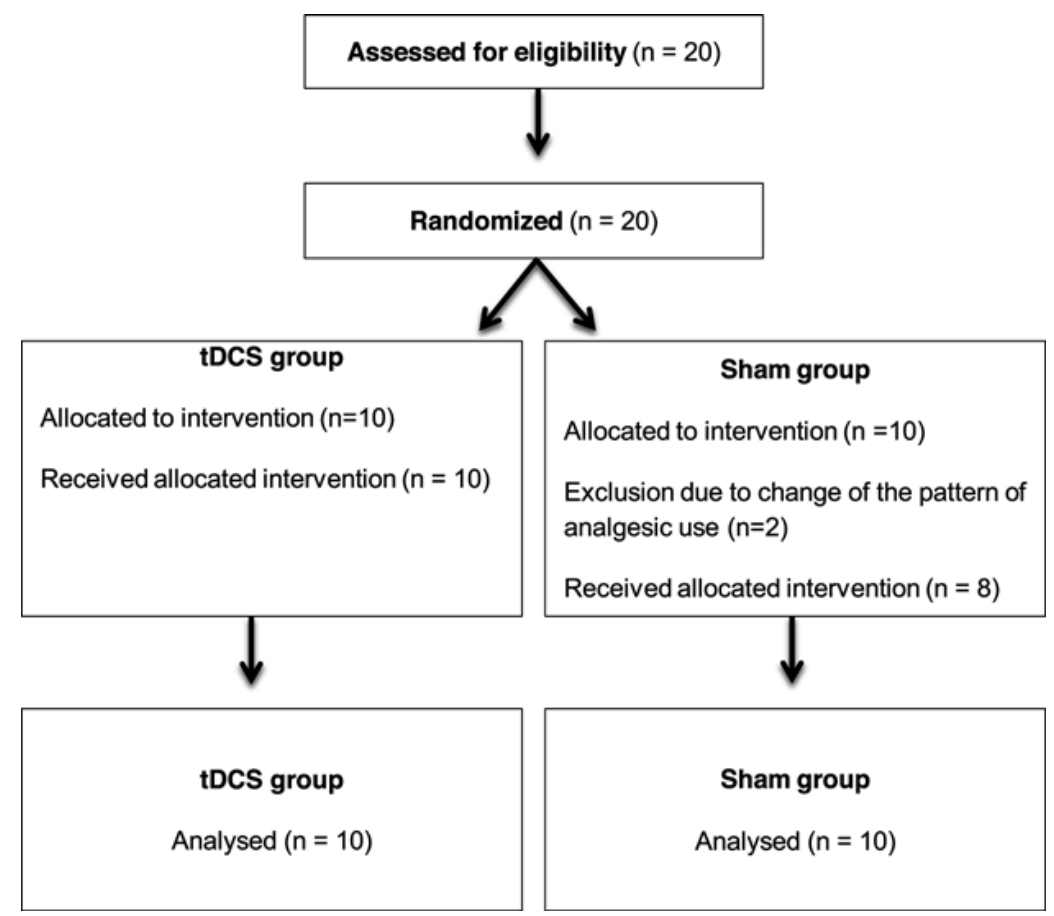

FIGURE 1. Flowchart of patient eligibility and randomization to transcranial direct current stimulation (tDCS) and sham groups. 


\begin{tabular}{lccc}
\hline \multicolumn{3}{l}{ TABLE 1. Baseline Characteristics of the Patients Infected With } \\
HTLV-1 and Submitted to tDCS or Sham & & \\
\hline Characteristics & tDCS Group & Sham Group & \\
(n= 18) & $(\mathbf{n}=\mathbf{1 0})$ & $(\mathbf{n}=\mathbf{1 0})$ & $\boldsymbol{P}$ \\
\hline Sex, female & $8(80)$ & $7(70)$ & 1.0 \\
Age (y) & $47.8 \pm 11.6$ & $56.1 \pm 14.0$ & 0.1 \\
Use of walking device & $5(50)$ & $4(40)$ & 0.7 \\
Pain location & & & 1.0 \\
$\quad$ Lower back & $9(90)$ & $8(80)$ & \\
$\quad$ Lower limbs & $1(10)$ & $2(20)$ & \\
Type of pain & & & 0.6 \\
Neuropathic & $6(60)$ & $7(70)$ & \\
Nociceptive & $4(40)$ & $3(30)$ & \\
Pain intensity* & $5.6 \pm 1.3$ & $5.95 \pm 1.1$ & 0.6 \\
Depression & $8(80)$ & $6(60)$ & 0.6 \\
Pressure pain threshold & $6.4 \pm 2.8$ & $5.9 \pm 2.0$ & 0.7 \\
TUG & $18.5(11.5-28.1)$ & $17.54(10.9-27.0)$ & 0.5 \\
SF-MPQ & $9.2 \pm 2.9$ & $10.2 \pm 2.9$ & 0.4 \\
Total score & $4.1 \pm 1.7$ & $5.2 \pm 2.3$ & 0.2 \\
Sensory aspect & $3.5(2.7-5.0)$ & $3.5(2.8-4.3)$ & 0.9 \\
Affective aspect & $2(1.0-2.0)$ & $1.5(1.0-2.0)$ & 0.7 \\
$\quad$ Evaluative aspect & & & \\
Quality of life & $21 \pm 13.8$ & $25.5 \pm 22.9$ & 0.6 \\
Physical functioning & $21 \pm(0-12.5)$ & $0(0-6.2)$ & 1.0 \\
Role-physical & $32.2 \pm 18.2$ & $39 \pm 24.7$ & 0.5 \\
Bodily pain & $43 \pm 19.6$ & $42.5 \pm 19.0$ & 1.0 \\
General health & $37.5(13.7-40)$ & $30(22.5-51.2)$ & 0.8 \\
Vitality & $51 \pm 21.0$ & $50 \pm 31.1$ & 0.9 \\
Social functioning & $0(0-41.2)$ & $0(0-8.2)$ & 0.4 \\
Role-emotional & $54 \pm 26.3$ & $48 \pm 24.8$ & 0.6 \\
Mental health & & & \\
\hline & & & \\
\hline
\end{tabular}

Data are shown as $\mathrm{n}(\%)$, mean $\pm \mathrm{SD}$, or median (25th to 75 th percentile) $\chi^{2}$ or Fisher exact test performed for the analysis of the categorical variables and the Student $t$ test for independent groups or Mann-Whitney test for continuous variables.

*Pain intensity indexed as a 10-point Visual Analog Scale $(0=$ "no pain" and $10=$ "worst possible pain").

tDCS indicates transcranial direct current stimulation; TUG, Timed Up and Go task; SF-MPQ, Short Form McGill Pain Questionnaire.

difference from baseline VAS scores: D1 1.56, $P=0.3$; D2 2.09, $P=0.048 ;$ D3 3.07, $P=0.052 ;$ D4 3.16, $P=0.03$; D5 $2.86, P=0.04)$.

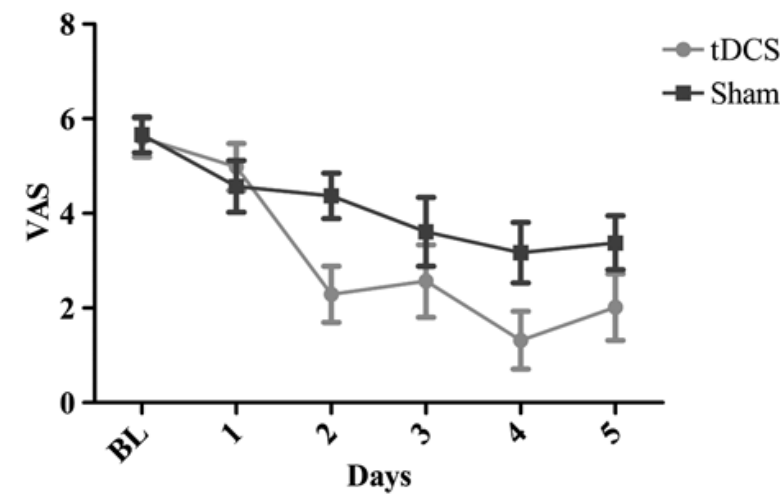

FIGURE 2. Daily comparison of pain intensity, as indexed by the Visual Analog Scale (VAS) (mean $\pm S D$ ), between treatment groups (active vs. sham): baseline $5.6 \pm 1.29$ versus $5.66 \pm 1.19$, $P=0.92 ; \quad D 1 \quad 4.98 \pm 1.56$ versus $4.57 \pm 1.74, \quad P=0.59 ; \quad D 2$ $2.29 \pm 1.87$ versus $4.37 \pm 1.52, P=0.01 ; \mathrm{D} 32.57 \pm 2.43$ versus $3.61 \pm 2.29, \quad P=0.34 ;$ D4 $1.32 \pm 1.93$ versus $3.17 \pm 2.02$, $P=0.051 ;$ D5 $2.03 \pm 2.23$ versus $3.38 \pm 1.80, P=0.15$. Analyses were performed using the Student $t$ test for independent groups. tDCS indicates transcranial direct current stimulation.

Overall, the mean percentage of VAS pain intensity reduction after 5 days of treatment was $62 \% \pm 40 \%$ in the tDCS group and $40.4 \% \pm 30.9 \%$ in the sham group $\left(\chi^{2}\right.$, $P=0.20)$. From these results, post hoc tests were carried out to elucidate the analgesic effects found in both the groups. Considering a $30 \%$ pain reduction cutoff, there were $8(80 \%)$ responders in the tDCS group and $7(70 \%)$ in the sham group (Fisher exact test, $P=1.00$ ). When this cutoff was raised to $50 \%$, the distribution changed to 8 $(80 \%)$ responders in the tDCS group and $3(30 \%)$ in the sham group (Fisher exact test, $P=0.03$ ).

The effects of the intervention on PPT, pain aspects, and TUG are depicted in Table 3. Although there were significant differences in the intragroup analysis for most of these variables, similar behavior could not be found for between-group comparisons.

\begin{tabular}{|c|c|c|c|c|}
\hline $\begin{array}{l}\text { Adverse Events } \\
(n=20)\end{array}$ & $\begin{array}{l}\text { tDCS Group } \\
\quad(n=10)\end{array}$ & $\begin{array}{l}\text { Sham Group } \\
\quad(n=10)\end{array}$ & Total & $P^{*}$ \\
\hline Tingling $\dagger$ & $9(90)$ & $7(70)$ & $16(80)$ & 0.58 \\
\hline Pulsing $\dagger$ & $2(20)$ & $3(30)$ & $5(25)$ & 1.00 \\
\hline Pricking $\dagger$ & $4(4)$ & 0 & $4(20)$ & 0.09 \\
\hline Pressing $\dagger$ & 0 & $1(10)$ & $1(5)$ & 1.00 \\
\hline Pinching $\dagger$ & 0 & $1(10)$ & $1(5)$ & 1.00 \\
\hline Burning $\dagger$ & $2(20)$ & 0 & $2(10)$ & 0.47 \\
\hline Somnolence & $2(20)$ & 0 & $2(10)$ & 0.47 \\
\hline Hyperemia $\ddagger$ & $1(10)$ & 0 & $1(5)$ & 1.00 \\
\hline \multicolumn{5}{|c|}{$\begin{array}{l}\text { Data are shown as } \mathrm{n}(\%) \text {. } \\
{ }^{*} \chi^{2} \text { test. } \\
¥ \text { These sensations were restricted to the area underneath the electrodes or the surrounding area. } \\
\text { \$The hyperemia was noticed by the researchers delivering the stimulation at the end of the first session of } \\
\text { atment. The hyperemia was restricted to the area under the cathode, lasting } 1-2 \text { minutes, resolving without any } \\
\text { ecific intervention. The patient also felt a pulsing sensation from the } 15 \text { th to the } 20 \text { th minute of that stimulation. } \\
\text { is patient only felt a transient tingling sensation on the second, third, and fifth sessions of treatment. } \\
\text { tDCS indicates transcranial direct current stimulation. }\end{array}$} \\
\hline
\end{tabular}


TABLE 3. Comparison of Pressure Pain Threshold, Pain Aspects, and Timed Up and Go at Baseline and After 5 Days of tDCS or Sham Treatment in Patients Infected With HTLV-1

\begin{tabular}{|c|c|c|c|c|c|}
\hline \multirow[b]{2}{*}{$\begin{array}{l}\text { Variables } \\
(\mathbf{n}=\mathbf{2 0})\end{array}$} & \multicolumn{5}{|c|}{ Groups } \\
\hline & $\begin{array}{c}\text { tDCS } \\
(n=10)\end{array}$ & Intragroup & $\begin{array}{c}\text { Sham } \\
(\mathbf{n}) \dagger\end{array}$ & Intragroup $(\boldsymbol{P})^{*}$ & Intergroup $(P)^{* *}$ \\
\hline Visual Analog Scale & & 0.001 & & 0.004 & NA $\|$ \\
\hline Before & $5.6 \pm 1.3$ & & $5.7 \pm 1.2$ & & \\
\hline Day 1 & $5.0 \pm 1.6$ & & $4.6 \pm 1.7$ & & \\
\hline Day 2 & $2.3 \pm 1.9$ & & $4.4 \pm 1.5$ & & \\
\hline Day 3 & $2.6 \pm 2.4$ & & $3.6 \pm 2.3$ & & \\
\hline Day 4 & $1.3 \pm 1.9$ & & $3.2 \pm 2.0$ & & \\
\hline Day 5 & $2.0 \pm 2.2$ & & $3.4 \pm 1.8$ & & \\
\hline Pressure Pain threshold & & 0.400 & & 0.57 & \\
\hline Before: & $6.4 \pm 2.8$ & & $5.7 \pm 2.1$ & & 0.58 \\
\hline After§ & $6.2 \pm 2.6$ & & $5.4 \pm 1.9$ & & 0.5 \\
\hline Total SF-MPQ score & & 0.002 & & 0.001 & \\
\hline Before & $9.2 \pm 2.9$ & & $10.9 \pm 2.8$ & & 0.23 \\
\hline After§ & $3.9 \pm 3.3$ & & $4.4 \pm 2.8$ & & 0.75 \\
\hline Sensory aspect & & 0.030 & & 0.001 & \\
\hline Beforeł & $4.1 \pm 1.7$ & & $5.8 \pm 2.0$ & & 0.07 \\
\hline After§ & $1.9 \pm 1.6$ & & $1.9 \pm 1.1$ & & 0.93 \\
\hline Affective aspect & & 0.007 & & 0.017 & \\
\hline Before & $3.5(2.8-5.0)$ & & $4.0(3.0-4.75)$ & & 0.75 \\
\hline After§ & $1.0(0-3.0)$ & & $1.5(0-3.0)$ & & 0.85 \\
\hline Evaluative aspect & & 0.020 & & 0.10 & \\
\hline Beforet: & $2.0(1.0-2.0)$ & & $1.5(1.0-2.0)$ & & 0.68 \\
\hline After§ & $0.5(0-1.0)$ & & $1(0.25-1.75)$ & & 0.25 \\
\hline Timed Up and Go & & 0.022 & & 0.039 & \\
\hline Before & $18.5(11.5-28.0)$ & & $21(10.7-27.9)$ & & 0.86 \\
\hline After§ & $14.3(10.920 .9)$ & & $17.8(10-22.4)$ & & 0.8 \\
\hline
\end{tabular}

Data are shown as mean \pm SD or median (25th to 75 th percentile)

Student $t$ test for paired samples or Wilcoxon signed-rank test was used for the comparison between the baseline state and after 5 days of treatment within each group, except VAS values, which were evaluated by repeated-measures ANOVA.

Bold values represent $P<0.05$.

*Paired $t$-test or Mann-Whitney, $P<0.05$.

**Unpaired $t$-test of Wilcoxon, $P<0.05$.

$t \mathrm{n}=10$ for Visual Analog Scale; $\mathrm{n}=8$ other variables.

$₫$ Baseline values.

$\S$ Values after 5 days of treatment.

$\| \mathrm{NA}=$ not applicable. Intergroup comparisons were made by the mixed model of repeated-measures ANOVA, presented in Figures 2 and 3.

HTLV-1 indicates human T-lymphotropic virus type I; SF-MPQ, Short Form McGill Pain Questionnaire; tDCS, transcranial direct current stimulation.

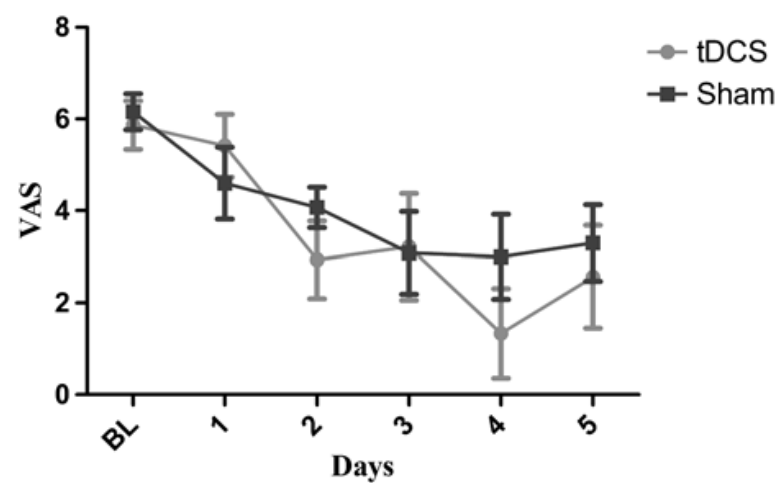

FIGURE 3. Subgroup comparison of daily pain intensity, as indexed by the Visual Analog Scale (VAS) (mean \pm SD), between stimulation groups (active vs. sham) considering only patients with neuropathic pain: baseline $5.87 \pm 1.29$ versus $6.16 \pm 1.04$, $P=0.6$; D1 $5.42 \pm 1.69$ versus $4.6 \pm 2.07, P=0.5$; D2 $2.93 \pm 2.07$ versus $4.07 \pm 1.16, P=0.2$; D3 $3.22 \pm 2.84$ versus $3.09 \pm 2.37$, $P=0.9$; D4 $1.33 \pm 2.39$ versus $3 \pm 2.44, P=0.2 ;$ D5 $2.57 \pm 2.73$ versus $3.3 \pm 2.19, P=0.6$. Analyses were performed using the Student $t$ test for independent groups. tDCS indicates transcranial direct current stimulation.

\section{DISCUSSION}

The present study demonstrated that tDCS applied in patients with HTLV-1 infection and chronic pain resulted in a similar analgesic effect to sham treatment, with no difference between them when $30 \%$ decrease in pain was considered. However, this was observed in a greater number of patients who presented $\geq 50 \%$ reduction in pain intensity in the tDCS group. Both tDCS and sham treatment groups presented a high incidence of AEs, especially during the first moments of treatment, and this occurrence may have accounted for the presence of clinically important placebo analgesia. In addition, there was an improvement in sensory-discriminative and affective-motivational aspects of pain and gait performance in both treatment groups. Nevertheless, PPT did not change in either group.

The main hypothesis of this study was that the tDCS treatment would provide significant pain intensity reduction in patients with HTLV-1 infection and chronic low back and/or lower limbs pain, which was not supported by our findings. Although the study group that underwent active tDCS intervention experienced significant analgesia, similar effects were observed in sham group. Analogous results were demonstrated for patients who presented chronic pain with predominant neuropathic components. 
These findings might be partially explained by the occurrence of important placebo analgesia.

The most probable explanation for the occurrence of a placebo effect of this magnitude might be related to the elevated incidence of AEs in the study, which could have led the majority of the patients to perceive that they were being actively stimulated. This could have affected the perception of treatment and expectations regarding the outcomes of the intervention. ${ }^{36}$ In turn, the increased rate of AEs could be attributable to the high electric current density, caused by the combination of smaller sized electrodes $\left(25 \mathrm{~cm}^{2}\right)$ and $2 \mathrm{~mA}$ current used in the protocol. In addition, as patients in our academic center were not previously exposed to experimental studies involving tDCS, their expectations with the procedure might have been higher compared with participants in other studies, thus increasing placebo effect.

One important point is blinding of tDCS. Although recent studies in pain-free participants have questioned the blinding efficacy of tDCS, ${ }^{37}$ it is important to note that blinding is different for naïve participants ${ }^{38}$ and in clinical trials - such as this one. In fact, a recent clinical trial showed that blinding effectiveness of tDCS was similar to placebo pills - the gold standard of blinding method. ${ }^{39}$ Also, given the large placebo response, it is conceivable to consider that blinding was at least partially effective. Recent experiments are testing the use of topically applied anesthetics before tDCS as to decrease minimal pain and itching associated with the effects of the electrical current, thus to decrease placebo response. ${ }^{40}$

An issue that merit further investigation is the nature of pain in HTLV-1 infection. Previous studies have shown that a high proportion of these patients have chronic low back and/or lower limbs pain of mixed origin. ${ }^{6-8}$ In general, there is no overall preponderance of neuropathic over nociceptive pain; nevertheless, when superimposed, neuropathic pain characteristics are usually more disabling. ${ }^{7-9}$ Although the mechanisms of chronic pain in this setting are not clear, they might be related to spinal cord inflammation, peripheral neuropathy, or mechanical disturbances due to lower limbs spasticity. ${ }^{6}$ In addition, as it has been demonstrated in other chronic low back pain syndromes, ${ }^{41}$ supraspinal neuroplastic changes might also take part in chronic pain mechanisms in HTLV-1 infection. Favoring a role of musculoskeletal imbalances and deconditioning as associated factors in this setting, our group has shown that 2 different exercise interventions had significant analgesic effects in patients with chronic low back pain infected with HTLV-1. ${ }^{42,43}$ If mechanical factors play a major role in this condition, perhaps the parameters of stimulation could be changed to decrease spasticity and improve motor control.

Previous studies have shown that anodal tDCS over the M1 results in improvement of motor performance. ${ }^{20,44}$ Therefore, it was expected that tDCS would improve gait performance in the present study. However, as both groups demonstrated improvement in this task, as well as in VAS pain scores, we hypothesize that gait performance improvement was not directly caused by tDCS. Analgesia, or even the nonanalgesic placebo effect, might explain the improvement in gait.

Some limitations of this study should be regarded. The use of drugs that have direct effects over the central nervous system was not controlled. This could account for some of the heterogeneity observed in the effects of the tDCS, as some of these substances can interfere with the neuromodulatory properties of the intervention. ${ }^{34}$ The use of smaller electrodes in the present protocol might cause difficulties to compare the results of this investigation to previous studies evaluating the effects of tDCS in patients with chronic pain. At last, placebo analgesia acted as a confounding factor for the association between tDCS and decrease in VAS pain scores, as previously discussed.

In the context of this preliminary, exploratory study with a small sample size, our findings suggest that there is no difference between sham and active anodal tDCS over M1 with $2 \mathrm{~mA}$ for 20 minutes for 5 consecutive days for reducing pain intensity in HTLV-1-infected patients with chronic low back and/or lower limbs pain. Further, studies should optimize parameters of stimulation to decrease the rate of AEs associated with the intervention and so minimize the occurrence of placebo analgesia.

\section{ACKNOWLEDGMENT}

The authors thank Mrs. Sonia Rangel, HTLV Center, Bahia School of Medicine and Human Health, Salvador, $B A /$ Brazil for secretarial support.

\section{REFERENCES}

1. Poiesz BJ, Ruscetti FW, Gazdar AF, et al. Detection and isolation of type $\mathrm{C}$ retrovirus particles from fresh and cultured lymphocytes of a patient with cutaneous T-cell lymphoma. Proc Natl Acad Sci USA. 1908;77:7415-7419.

2. Morgan OS, Rodgers-Johnson P, Mora C, et al. HTLV-1 and polymyositis in Jamaica. Lancet. 1989;2:1184-1187.

3. Mochizuki M, Ono A, Ikeda E, et al. HTLV-I uveitis. J Acquir Immune Defic Syndr Hum Retrovirol. 1996;13(suppl 1): S50-S56.

4. Nishioka K, Maruyama I, Sato K, et al. Chronic inflammatory arthropathy associated with HTLV-I. Lancet. 1989;1:399-456.

5. La Grenade L. HTLV-I associated infective dermatitis: past, present and future. J Acquir Immune Defic Syndr Hum Retrovirol. 1996;13(suppl 1):S46-S49.

6. Castro-Costa CM, Campos-Araújo Ade Q, Câmara CC, et al. Pain in tropical spastic paraparesis/HTLV-I associated myelopathy patients. Arq Neuropsiquiatr. 2009;67(3B):866-870.

7. Netto EC, Brites C. Characteristics of chronic pain and its impact on quality of life of patients with HTLV-1-associated myelopathy/tropical spastic paraparesis (HAM/TSP). Clin J Pain. 2011;27:131-135.

8. Tavares Ir, Franzoi AC, Araújo AQ. Low-back pain in HTLVI-associated myelopathy/tropical spastic paraparesis: nociceptive or neuropathic? Spinal Cord. 2010;48:134-137.

9. Martins JV, Baptista AF, Araújo Ade Q. Quality of life in patients with HTLV-I associated myelopathy/tropical spastic paraparesis. Arq Neuropsiquiat. 2012;70:257-261.

10. Croda MG, de Oliveira AC, Vergara MP, et al. Corticosteroid therapy in TSP/HAM patients: the results from a 10 years open cohort. J Neurol Sci. 2008;269:133-137.

11. Nitsche MA, Paulus W. Sustained excitability elevations induced by transcranial de motor cortex stimulation in humans. Neurology. 2001;57:1899-1901.

12. Nitsche MA, Fricke K, Henschke U, et al. Pharmacological modulation of cortical excitability shifts induced by transcranial direct current stimulation in humans. J Physiol. 2003; 553(pt 1):293-301.

13. Nitsche MA, Cohen LG, Wassermann EM, et al. Transcranial direct current stimulation: state of the art. Brain Stimul. 2008; 1:206-223.

14. Jang SH, Ahn SH, Byun WM, et al. The effect of transcranial direct current stimulation on the cortical activation by motor 
task in the human brain: an fMRI study. Neurosci Lett. 2009;460:117-120.

15. Fregni F, Boggio PS, Lima MC, et al. A sham-controlled, phase II trial of transcranial direct current stimulation for the treatment of central pain in traumatic spinal cord injury. Pain. 2006;122:197-209.

16. Mori F, Codeca C, Kusayanagi H, et al. Effects of anodal transcranial direct current stimulation on chronic neuropathic pain in patients with multiple sclerosis. J Pain. 2010;11: 436-442.

17. Fregni F, Gimenes R, Valle AC, et al. A randomized, sham controlled, proof of principle study of transcranial direct current stimulation for the treatment of pain in fibromyalgia. Arthritis Rheum. 2006;54:3988-3998.

18. Mendonca ME, Santana MB, Baptista AF, et al. Transcranial DC stimulation in fibromyalgia: optimized cortical target supported by high-resolution computational models. J Pain. 2011;12:610-617.

19. DaSilva AF, Volz MS, Bikson M, et al. Electrode positioning and montage in transcranial direct current stimulation. $J$ Vis Exp [serial online]. 2011;51:e2744.

20. Bastani A, Jaberzadeh S. Does anodal trancranial direct current stimulation enhance excitability of the motor cortex and motor function in healthy individuals and subjects with stroke: a systematic review and meta-analysis. Clin Neurophysiol. 2012;123:644-657.

21. Kincses TZ, Antal A, Nitsche MA, et al. Facilitation of probabilistic classification learning by transcranial direct current stimulation of the prefrontal cortex in the human. Neuropsychologia. 2004;42:113-117.

22. Antal A, Terney D, Kühnl S, et al. Anodal transcranial direct current stimulation of the motor cortex ameliorates chronic pain and reduces short intracortical inhibition. $J$ Pain Symptom Manage. 2010;39:890-903.

23. Antal A, Varga ET, Nitsche MA, et al. Direct current stimulation over MT + /V5 modulates motion after-effect in humans. Neuroreport. 2004;15:2491-2494, 15.

24. Lang N, Nitsche MA, Paulus W, et al. Effects of transcranial direct current stimulation over the human motor cortex on corticospinal and transcallosal excitability. Exp Brain Res. 2004;156:439-443.

25. Valle A, Roizenblatt S, Botte S, et al. Efficacy of anodal transcranial direct current stimulation (tDCS) for the treatment of fibromyalgia: results of a randomized, shamcontrolled longitudinal clinical trial. J Pain Manag. 2009;2: 353-361.

26. O'Connell NE, Wand BM, Marston L, et al. Non-invasive brain stimulation techniques for chronic pain. A report of a Cochrane systematic review and meta-analysis. Eur J Phys Rehabil Med. 2011;47:309-326.

27. Ferreira KA, Andrade DC, Teixeira MJ. Development and validation of a Brazilian version of the Short-Form McGill Pain Questionnaire (SF-MPQ). Pain Manag Nurs. 2011;13:1-10.

28. Santos JG, Brito JO, Andrade DC, et al. Translation to Portuguese and validation of the Douleur Neuropathique 4 questionnaire. J Pain. 2010;11:484-490.
29. Fischer AA. Pressure algometry over normal muscles. Standard values, validity and reproducibility of pressure threshold. Pain. 1987;30:115-126.

30. Podsiadlo D, Richardson S. The timed "Up \&Go": a test of basic functional mobility for frail elderly persons. J Am Geriatr Soc. 1991;39:142-148.

31. Ciconelli RM, Ferraz MB, Santos W, et al. BrazilianPortuguese version of SF-36: a reliable and valid quality of life outcome measure. Rev Bras Reumatol. 1999;39:143-150.

32. Sheehan DV, Lecrubier Y, Sheehan KH, et al. The MiniInternational Neuropsychiatric Interview (M.I.N.I.): the development and validation of a structured diagnostic psychiatric interview for DSM-IV and ICD-10. J Clin Psychiatr. 1998; 59(suppl 20):22-23.

33. Homan RW, Herman J, Purdy P. Cerebral location of international 10-20 system electrode placement. Electroencephalogr Clin Neurophysiol. 1987;66:376-382.

34. Zaghi S, Thiele B, Pimentel D, et al. Assessment and treatment of pain with non-invasive cortical stimulation. Restor Neurol Neurosci. 2011;29:439-451.

35. Velasco MV, Dias TC, Freitas AZ, et al. Hair fiber characteristics and methods to evaluate hair physical and mechanical properties. Braz J Pharm Sci. 2009;45:153-162.

36. Colloca L, Klinger R, Flor H, et al. Placebo analgesia: psychological and neurobiological mechanisms. Pain. 2013; 154:511-514.

37. O'Connell NE, Cossar J, Marston L, et al. Rethinking clinical of transcranial direct current stimulation: participant and assessor blinding is inadequate at intensities of $2 \mathrm{~mA}$. PLoS One. 2012;7:e47514.

38. Ambrus GG, Al-Moyed H, Chaieb L, et al. The fade-in-short stimulation - fade out approach to sham tDCS - reliable at $1 \mathrm{~mA}$ for naive and experienced subjects, but not investigators. Brain Stimul. 2012;5:499-504.

39. Brunoni AR, Valiengo L, Baccaro A, et al. The sertraline vs. electrical current therapy for treating depression study: results from a factorial, randomized, controlled trial. JAMA Psychiatry. 2013;70:383-391.

40. McFadden JL, Borckardt JJ, Goerge MS, et al. Reducing procedural pain and discomfort associated with transcranial direct current stimulation. Brain Stimul. 2011;4:38-42.

41. Baliki MN, Schnitzer TJ, Bauer WR, et al. Brain morphological signatures for chronic pain. PLoS One. 2011;6:e26010.

42. Borges J, Baptista AF, Santana N, et al. Pilates exercises improve low back pain and quality of life in patients with HTLV-1 virus: a randomized crossover clinical trial. J Bodyw Mov Ther. 2013; (In press).

43. Figueiredo Neto I, Mendonça RP, Nascimento CA, et al. Fortalecimento muscular em pacientes com HTLV-1 e sua influência no desempenho functional: Um estudo piloto [Muscle strengthening in patients with HTLV-1 and its influence on functional performance: a pilot study]. Rev Pesq Fisio. 2012;2:143-155.

44. Hummel F, Celnik P, Giraux P, et al. Effects of non-invasive cortical stimulation on skilled motor function in chronic stroke. Brain. 2005;128(pt 3):490-499. 